\title{
ESTUDIO ANATÓMICO DEL TRONCO VENOSO GASTROCÓLICO
}

\author{
Andres Pouy, Juan Cabrera, Gustavo Armand Ugón \\ Departamento de Anatomia, Facultad de Medicina, Universidad de la Republica,
Montevideo, Uruguay
}

\section{RESUMEN}

Introducción: El tronco venoso gastrocólico, según lo descrito por Henle en 1868 se forma de la confluencia de las venas gastroepiploica derecha, cólica derecha y pancreático duodenal anteroinferior. Su localización y conocimiento anatómico es de importancia quirúrgica en la movilización duodeno pancreática y coloepiploica. Material y métodos: Se utilizaron 13 cadáveres formolados, adultos, de ambos sexos. Se registró: formación del tronco venoso gastrocólico, calibre de los afluentes y del tronco, distancias entre: cuello del páncreas y borde superior del duodeno III, vena mesentérica superior a duodeno II. Se topografió el tronco venoso en relación a las estructuras mencionadas. Se registró largo del tronco, distancia al borde superior del duodeno III y al borde inferior del cuello de páncreas. Resultados: la conformación más frecuente fue por confluencia de las venas cólica derecha, gastroepiploica derecha y pancreático duodenal anteroinferior. El calibre medio del tronco venoso fue $5,65 \mathrm{~mm} \quad(3,3 \mathrm{~mm}-10 \mathrm{~mm})$. La distancia media entre duodeno III y cuello de páncreas fue de $31,34 \mathrm{~mm}(13,2 \mathrm{~mm}-51 \mathrm{~mm})$. La distancia media entre vena mesentérica superior y duodeno II fue de $34,23 \mathrm{~mm}(23,8 \mathrm{~mm}-45,7 \mathrm{~mm})$. El largo medio del tronco venoso fue $9,43 \mathrm{~mm}(3,2 \mathrm{~mm}-16,3 \mathrm{~mm})$.Conclusión: se constató, en la mayoría de los casos, que la confluencia de formación del tronco venoso se daba según lo descrito clásicamente. Este se situó más frecuentemente de disposición oblicua hacia abajo y adentro, y en el cuadrante ínfero-interno respecto al cuadrilátero dado por una línea vertical desde cuello del páncreas a duodeno III y una horizontal desde duodeno II hasta la vena mesentérica superior.

Palabras clave: venas pancreáticas; venas cólicas; colectomía derecha; pancreatectomía.

\section{ABSTRACT}

Introduction: the gastrocolic venous trunk, as described by Henle in 1868, is formed from the confluence of the right gastroepiploic, right colic, and anteroinferior duodenal pancreatic veins. Its location and anatomical knowledge is of surgical importance in pancreatic and colo-epiploic duodenal mobilization. Material and methods: 13 cadavers were used, adults, of both sexes. The following were recorded: gastrocolic venous trunk formation, caliber of tributaries and trunk, distances between: neck of pancreas and upper border of duodenum III, superior mesenteric vein to duodenum II. The venous trunk was topographed in relation to the mentioned structures. The length of the trunk was recorded, distance to the upper border of the duodenum III and to the lower border of the pancreas neck. Results: the most frequent conformation was by confluence of the right colic, right gastroepiploic and duodenal anteroinferior pancreatic veins. The average caliber of the venous trunk was $5.65 \mathrm{~mm}(3.3 \mathrm{~mm}$ $10 \mathrm{~mm})$. The mean distance between duodenum III and pancreatic neck was $31.34 \mathrm{~mm}(13.2 \mathrm{~mm}-51 \mathrm{~mm})$. The mean distance between superior mesenteric vein and duodenum II was $34.23 \mathrm{~mm}$ (23.8mm-45.7mm). The mean length of the venous trunk was $9.43 \mathrm{~mm}(3.2 \mathrm{~mm}$ $16.3 \mathrm{~mm}$ ). Conclusion: it was found, in most cases, that the confluence of venous trunk formation was given according to the classically described. This was located more frequently with an oblique disposition downwards and inwards, and in the inferior-internal quadrant with respect to the quadrilateral given by a vertical line from the neck of the pancreas to duodenum III and a horizontal line from duodenum II to the superior mesenteric vein.

Key words: pancreatic veins; colic veins; right colectomy; pancreatectomy.
* Correspondencia a: Juan A. Cabrera
cab0994row31@gmail.com

Recibido: 11 de Agosto, 2019. Revisado: 6 de Setiembre, 2019. Aceptado: 11 de Octubre, 2019. 


\section{INTRODUCCIÓN}

El tronco venoso gastrocólico (TG) fue descrito por Henle en 1868. En su descripción original Henle (1868) lo define como el tronco venoso formado por la unión de la vena gastroepiploica derecha y la vena cólica derecha (Bergamaschi et al, 2008). En 1912 Descomps y De Lalaubie (1912) reportan que en algunos casos confluye también la vena pancreático-duodenal anterosuperior. En los tratados clásicos de anatomía actualmente recomendados para los estudiantes de grado, la conformación del tronco gastrocólico es breve, poco clara y discordante. El TG se localiza en relación a la cara anterior de la cabeza del páncreas y se aboca en el borde derecho de la vena mesentérica superior por debajo del cuello del páncreas. Por su localización y rol en el drenaje venoso de varios órganos como el colon derecho,el estómago y parte del duodeno y el páncreas, tiene gran relevancia quirúrgica, ya que múltiples procedimientos de uso frecuente en la cirugía digestiva pueden involucrarlo. Su lesión puede ser fuente de sangrado tanto durante una duodeno-pancreatectomía como durante una colectomía derecha, especialmente en la liberación del ángulo derecho del colon en la cirugía a cielo abierto, o en la disección mesial en la cirugía laparoscópica del colon derecho (Jin et al, 2006). Algunos autores han propuesto al tronco gastrocólico como límite para la disección de la vena mesentérica superior en la colectomía derecha oncológica (Gao y Lu, 2018). En la cirugía laparoscópica se pierde el sentido del tacto de forma directa. Esto conlleva a la necesidad de un mayor conocimiento anatómico de las estructuras y sus distintas variantes para minimizar los riesgos quirúrgicos. El objetivo del presente trabajo fue estudiar la anatomía del tronco venoso gastrocólico, sus afluentes y localizarlo respecto a las estructuras que lo rodean.

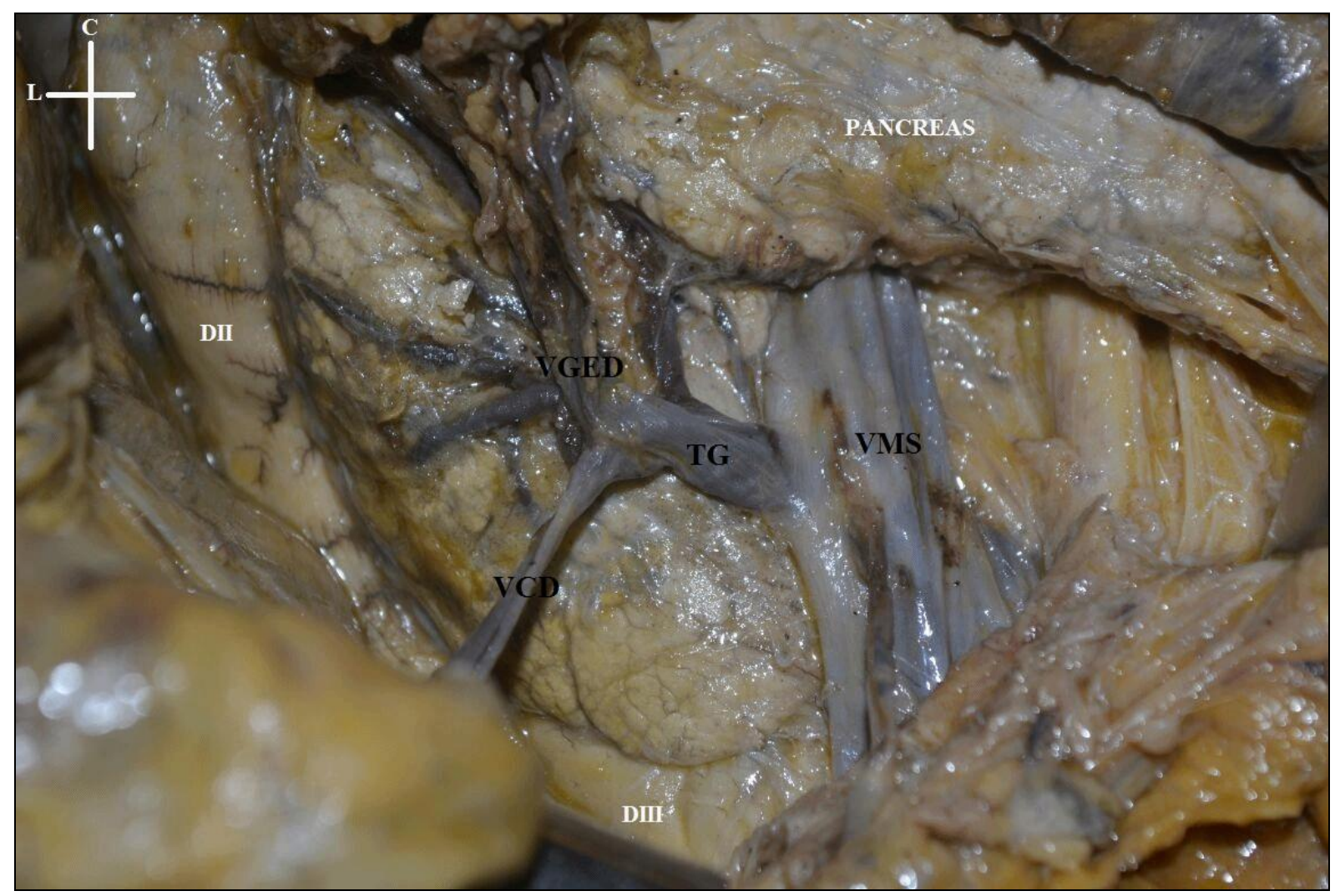

Figura 1- vista frontal del duodeno-páncreas. C: craneal, L: lateral. DII: segunda porción duodenal, DIII: tercera porción duodenal, CD: vena cólica derecha, VGED: vena gastroepiploica derecha, TG: tronco gastrocólico, VMS: vena mesentérica superior. 


\section{MATERIALES Y MÉTODO}

Se utilizaron 13 cadáveres adultos fijados con solución en base a formol $(\mathrm{N}=13)$. Se disecó la vena mesentérica superior (VMS) desde el borde superior de la tercera porción del duodeno (D III) hasta el borde inferior del cuello del páncreas, punto en el cual la VMS queda por detrás del mismo. Se definió al TG como aquel tronco venoso que desemboca a la derecha de la VMS en el segmento disecado y que estuviera formado por la confluencia de al menos 2 venas. Se registraron las venas que originaron el TG, su calibre, el calibre y el largo del TG. Se midió la distancia entre el borde derecho de la VMS al borde medial de la segunda porción del duodeno (D II) medido de forma horizontal desde el punto medio de la VMS en el segmento disecado. Se midió la distancia entre el TG y el borde superior de DIII, y entre el TG y el borde inferior del cuello del páncreas. Se definió un cuadrilátero delimitado por: borde inferior del cuello del páncreas (límite superior), borde superior de DIII (límite inferior), VMS (límite izquierdo) y borde medial de DII (límite derecho), se dividió dicho cuadrilátero en cuadrantes y se topografió el TG en dichos cuadrantes. Todas las medidas fueron tomadas con calibre digital.

\begin{tabular}{|l|l|}
\hline Venas & frecuencia de presentación $(\%)$ \\
\hline VCD + VGED & 2 casos $(15 \%)$ \\
\hline VCD + VGED + VPDAS & 5 casos $(38 \%)$ \\
\cline { 2 - 2 } VCD + VGED + VPDAI & 1 caso $(8 \%)$ \\
\hline VCD + VGED + VPDAS + VPDAI & 3 casos $(23 \%)$ \\
\cline { 2 - 2 } VCD + VGED + VPDAI + VCM & 2 casos $(15 \%)$ \\
\hline
\end{tabular}

Tabla 1- Formación del tronco venoso gastrocólico. VCD. Vena cólica derecha. VGED: vena gastroepiplóica dererecha. VPDAS: vena pancreáticoduodenal anterosuperior. VPDAI: vena pancreáticoduodenal anteroinferior. VCM: vena cólica media.

\section{RESULTADOS}

El TG se presentó en el total de cadáveres estudiados, 13 (100\%) casos (Figura 1); siendo su conformación de manera variable. La vena cólica derecha (VCD) formó parte de la confluencia en 13 (100\%) cadáveres. La vena gastroepiploica derecha (VGED) lo hizo en 12 $(92 \%)$ casos. La vena pancreático-duodenal anteroinferior (VPDAl) formó parte de la confluencia en 11 (85\%) casos. Otras venas se presentaron formando parte de la confluencia en $6(46 \%)$ casos, donde la vena cólica media (VCM) se presentó en $3(23 \%)$ casos; al igual que la vena pancreático-duodenal anterosuperior (VPDAS).

Encontramos que la formación del TG se presentó con las siguientes variantes: en 5 (38\%) casos se conformaba por la reunión de vena cólica derecha más vena gastroepiploica derecha más vena pancreaticoduodenal anterior e inferior; 3 (23\%) casos vena cólica derecha más vena gastroepiploica derecha más vena pancreático-duodenal anteroinferior + vena pancreático-duodenal anterosuperior; 2 (15\%) casos vena cólica derecha más vena gastro- epiploica derecha más vena pancreáticoduodenal anteroinferior más vena cólica media; 2 (15\%) casos vena cólica derecha más vena gastroepiploica derecha; y 1 (8\%) caso vena cólica derecha más vena pancreático-duodenal anteroinferior más vena gastroepiploica derecha (Tabla 1). El tipo de conformación del TG.

El calibre medio del TG fue de $5,6 \mathrm{~mm}$ y su largo promedio de $9,4 \mathrm{~mm}$. En cuanto a los calibres de los afluentes, la VCD se presentó con un calibre medio de $3,5 \mathrm{~mm}$. El calibre medio de la VGED fue de $4 \mathrm{~mm}$. El calibre medio de la VPDAI fue de $2,2 \mathrm{~mm}$.

La distancia media entre el abocamiento del TG en la vena mesentérica superior hasta el borde superior del DIII fue de 15,3mm, y desde su abocamiento en la VMS hasta el borde inferior del cuello de páncreas fue de $17,6 \mathrm{~mm}$.

En cuanto a la situación del TG, este se situó más frecuentemente en el cuadrante ínfero interno, 7 (53\%) casos.

En cuanto a la dirección del TG, desde su formación a su abocamiento en la VMS, este se dirigió hacia abajo y adentro en 5 (38\%) casos, de manera horizontal en $5(38 \%)$ casos, y hacia arriba y adentro en $3(24 \%)$ casos. 


\section{DISCUSIÓN}

Se encontró de forma constante el TG, dato que se correlaciona con lo hallado por Ignjatovicet al. (2004) en su estudio cadavérico, $100 \%(\mathrm{~N}=10)$ y Zhang et al. (1994) en su estudio radioanatómico, quien lo haya con una frecuencia del 94\% (51 en 54 casos). Sin embargo hay autores que lo describen en mucho menor frecuencia, como Lange et al.(2000) y Moriet al. (1992) que hallaron la presencia del TG en un $46 \% \quad(\mathrm{~N}=37)$, $y$ en un $48 \%(\mathrm{~N}=58)$ respectivamente. Para el segundo caso creemos que la diferencia es atribuible al método de registro utilizado, que fue tomográfico y en cortes gruesos no menores a $5 \mathrm{~mm}$. Teniendo en cuenta que, según las medidas registradas en el presente estudio, el calibre promedio del TG fue de 5,6 mm. Lo dicho es evidente en el propio estudio, en el cual la frecuencia del TG aumenta casi al doble comparando cortes de 5 y $10 \mathrm{~mm}$, siendo una clara limitante de la tomografía para el estudio del TG.

Por su parte la formación del TG ha sido variable según todos los autores consultados. Zhang et al. (1994) y Lange et al. (2000) describen un tronco venoso tripodal como presentación más frecuente, dato que se correlaciona con nuestros resultados. La presentación tripodal más frecuente según estos autores fue la confluencia de vena gastroepiploica derecha más vena cólica derecha más vena pancreático-duodenal anterosuperior, $18(35 \%)$ casos. A diferencia del presente trabajo en el cual participaba la vena pancreático-duodenal antero-inferior en vez de la vena pancreático-duodenal anterosuperior.

Dichos autores describen un tronco bipodal como segunda presentación en frecuencia, a diferencia de nuestros resultados, siendo el segundo en frecuencia la conformación cuatripodal. Creemos que dichas diferencias puedan deberse al tamaño de nuestra muestra.

En cuanto al estudio de las venas tributarias del tronco gastrocólico se encontró como las más constantes la vena cólica derecha y vena Gastroepiploica derecha.

La distancia hallada entre el abocamiento del TG y el borde superior de DIII no coincide con lo hallado por Jin et al. (2006) quien halló en su trabajo con cadáveres frescos una distancia media de $24 \mathrm{~mm}$. Creemos que esta diferencia puede estar relacionada a la técnica de conservación cadavérica utilizada en el material de investigación sobre el que realizamos nuestro trabajo.

Jin et al. (2006) también le describen al TG una dirección horizontal, dato que en parte se correlaciona con lo hallado, encontrando más frecuentemente una dirección horizontal pero también una dirección hacia abajo y adentro.

El presente trabajo constató la presencia del TG de manera constante, dándose su conformación en la mayoría de los casos según lo descrito clásicamente. Este estudio también situó más frecuentemente al TG en el cuadrante ínferointerno y de disposición oblicua hacia abajo y adentro, dato de utilidad para los variados procedimientos quirúrgicos que puedan involucrar al TG.

Se constató, en la mayoría de los casos, que la confluencia de formación del tronco venoso se daba según lo descrito clásicamente. Este se situó más frecuentemente en el cuadrante ínferointerno y de disposición oblicua hacia abajo y adentro.

\section{Conflicto de intereses}

Los autores declaran no tener conflicto de intereses.

\section{Financiamiento}

Los autores declaran no haber recibido ningún tipo de financiación económica por parte de terceros.

\section{Aprobación ética \\ No corresponde.}

\section{Consentimiento informado}

El Departamento de anatomía de la Facultad de Medicina, UdelaR, cuenta con el consentimiento informado de donantes cadavéricos para docencia e investigación.

\section{Contribuciones}

AP: disección cadavérica, recolección de datos y escritura del manuscrito. JC: disección cadavérica, recolección de datos y escritura del manuscrito. GAU: supervisación de la recolección de datos, escritura y revisión del manuscrito.

\section{BIBLIOGRAFÍA}

Bergamaschi R, Schochet E, Haughn C, Burke M, Reed JF, Arnaud JP. 2008. Standardized laparoscopic intracorporeal right colectomy for cancer: Short-term outcome in 111 unselected patients. Diseases of the Colon and Rectum 51: 1350-55.

Descomps $P$, De Lalaubie G. 1912. Les veines mésentériques. J Anat Physio Norm Pathol Homme Anim 48: 337-76. 
Jin G, Tuo H, Sugiyama M, Oki A, Abe N, Mori T, Masaki T,Atomi Y. 2006. Anatomic study of the superior right colic vein: its relevance to pancreatic and colonic surgery. Am J Surg 191: 100-03.

Gao Y, Lu Y. 2018. Variations of Gastrocolic Trunk of Henle and Its Significance in Gastrocolic Surgery. Gastroenterology Research and Practice 1: 1-8.

Henle J. 1868. Handbuch der Systematischen Anatomie des Menschen. 1ํEdición, Germany: Editorial Friedrich Vieweg und Sohn, pag 371.

Mori H, McGrath FP, Malone DE, Stevenson GW. 1992. The gastrocolic trunk and its tributaries: CT evaluation. Radiology 182: 871-77.

Ignjatovic D, Stimec B, Finjord T, Bergamaschi R. 2004. Venous anatomy of the right colon: threedimensional topographic mapping of the gastrocolic trunk of Henle. Tech Coloproctol8: 19-22.
Lange JF, Koppert S, Casper HJ, Eyck V, Kazemier G, Kleinrensink GJ, Godschalk M. 2000. The gastrocolic trunk of Henle in pancreatic surgery: an anatomo-clinical study. J Hepatobiliary Pancreat Surg 7: 401-03.

Zhang J, Rath AM, Boyer JC, Dumas JL, Menu Y, Chevrell JP. 1994. Radioanatomic study of the gastrocolie venous trunk. Surg Radiol Anat. 16: 413-418.

\section{AGRADECIMIENTOS}

Los autores manifiestan su reconocimiento a todos quienes en vida deciden donar su cuerpo a nuestra facultad para la docencia e investigación de la Anatomía. 